Economics Department

Temporal Disaggregation, Missing Observations, Outliers, and Forecasting:

A Unifying Non-Model Based Procedure

\title{
Massimiliano Marcellino
}

ECO No. $97 / 30$

\section{EUI WORKING PAPERS}

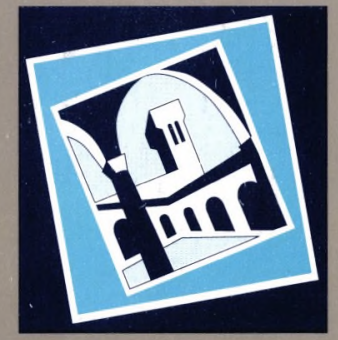


European University Institute

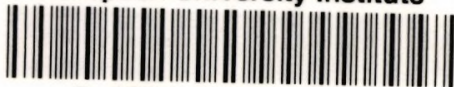

30001003554526 
EUROPEAN UNIVERSITY INSTITUTE, FLORENCE

ECONOMICS DEPARTMENT

EUI Working Paper ECO No. 97/30

Temporal Disaggregation, Missing Observations,

Outliers, and Forecasting:

A Unifying Non-Model Based Procedure

MASSIMILIANO MARCELLINO

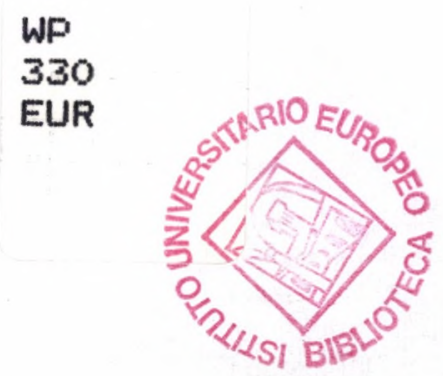

BADIA FIESOLANA, SAN DOMENICO (FI) 
All rights reserved.

No part of this paper may be reproduced in any form without permission of the author.

(C) Massimiliano Marcellino

Printed in Italy in December 1997

European University Institute

Badia Fiesolana

I - 50016 San Domenico (FI)

Italy 


\title{
Temporal Disaggregation, Missing Observations, Outliers, and Forecasting: A Unifying Non-Model Based Procedure
}

\author{
Massimiliano Marcellino*† \\ European University Institute
}

November 1997

\begin{abstract}
We suggest a simple non model based procedure to recover a time series from its temporally aggregated realizations. If additional assumptions on the underlying process are introduced, it is shown that the procedure is related to many of the former proposals in the literature. It can also be easily modified to deal with the estimation of missing observations and outliers, and with forecasting. Some important identification issues are finally discussed.
\end{abstract}

*I would like to thank Giampiero Gallo, Clive Granger, Soren Johansen, Marco Lippi, Grayham Mizon, Pravin Trivedi and participants at the 1996 Econometric Society European Meeting for useful comments. I alone am responsible for remaining errors.

†Correspondence to: Massimiliano Marcellino, Department of Economics, European University Institute, I-50016, San Domenico, Firenze, Italy. 


\section{Introduction}

There is quite often a mismatch between the frequency of data generation and that of data collection, the latter being usually rather lower than the former. This problem is particularly relevant in economics where, in general, at most monthly data are available for key variables such as GNP or unemployment. Moreover, only recently monthly data collection has systematically started and, usually, only annual data are available up to the ' 50 s and quarterly data up to the '70s. Hence, whenever the original realizations are of interest, they have to be recovered from the available temporally aggregated time series.

Thus, it is not surprising that the issue of temporal disaggregation of aggregated data has been extensively studied. At least five main approaches to the problem can be distinguished. The first method consists in recovering the disaggregated values by means of partial weighted averages of the aggregated ones, see e.g. Lisman and Sandee (1964). In the second method the disaggregated values are those which minimise a loss function under a compatibility constraint with aggregated data, see e.g. Boot et al. (1967), Cohen et al. (1971), Stram and Wei (1986). In the third method a similar problem is solved but it is assumed that a preliminary disaggregated series is available, so that the issue is how to best revise it in order for it to be compatible with aggregated data, see e.g. Denton (1971), Chow and Lin (1971), Fernandez (1981), and Litterman (1983). In the fourth method the assumption of a disaggregated ARIMA process is made, and an optimization problem similar to those in the second and third methods is solved, see e.g., respectively, Wei and Stram (1990) and Guerrero (1990). In the fifth method, the hypothesis of an ARIMA process is also maintained, but the disaggregated values are considered as missing observations, and their best estimators as such can be obtained, see e.g. Harvey and Pierse (1984), Kohn and Ansley (1986), Nijman and Palm (1986), and Gomez and Maravall (1994).

Our procedure belongs to the second method and assumes the mean squared disaggregation error, MSDE, as the loss function. This seems a reasonable choice because the MSDE is a widespread measure of the 
"goodness" of an estimator of the disaggregated data, and it is usually adopted to compare different procedures, see e.g. Chan (1993). In Section 2 we derive the linear estimator of the disaggregated data which minimizes this loss function and satisfies the aggregated data compatibility constraint. A modified procedure which keeps into account uncertainty about the disaggregated process is also introduced.

We make rather weak assumptions about the disaggregated process in solving the former problem. Actually, we only require the existence of moments up to order two. We then show that if some further assumptions are introduced, our proposal is related to other estimators in the second and fourth methods.

Some further issues such as the estimation of the unobservable series at different disaggregated frequencies, or with series at different aggregated frequency, the revision of preliminary estimates, and the derivation of a "disaggregator" which minimizes the generalized variance of the disaggregation errors are then discussed in Section 3.

In Section 4 we show that the procedure can be also applied to derive linear minimum MSDE estimators of missing observations and outliers, and to forecasting. We then relate our results to former findings in the literature on this topic, e.g., Whittle (1963), Chang et al. (1988).

In most of the aforementioned studies a substantial knowledge of the characteristics of the disaggregated process is assumed. Yet, usually, only aggregate information is available, and it is not sufficient to exactly identify the disaggregated characteristics of interest. In particular, to implement our procedure, as well as most of the other ones in the second and third methods, the variance covariance matrix of the disaggregated process is required. In Section 5 we show that it cannot be uniquely determined from that of the aggregated process, unless particular a priori restrictions are imposed. We suggest some conditions which are either only necessary or also sufficient for exact identification.

The identification problem is also present in the fourth and fifth methods for temporal disaggregation, because an infinite number of ARIMA processes are compatible with an aggregated one. The class of all admis- 
sible disaggregated ARIMA processes is derived in Section 6, a simple but rather stringent sufficient condition for the exact identification of one of them is proposed, while more general conditions are derived by Wei and Stram (1990). A simple example is then discussed to illustrate these issues. When there is uncertainty about the proper a priori restrictions to impose, a probabilistic statement about them, or about their implied variance matrices, can be made, and the modified procedure in Section 2 adopted.

Concluding remarks and a summary of the main results of the paper are proposed in Section 7.

\section{Estimation of disaggregated values}

Let us consider the zero mean stochastic process $x=\left\{x_{t}\right\}_{t=0}^{\infty}$ and assume that its realizations are subject to temporal aggregation before being observed. Hence, the observed values can be thought of as realizations of the process $y=\left\{y_{\tau}\right\}_{\tau=0}^{\infty}=\left\{\omega(L) x_{t k}\right\}_{t=1}^{\infty}$, where $k$ indicates the frequency of aggregation, $L$ is the lag operator, and $\omega(L)=\omega_{0}+\omega_{1} L+\ldots+\omega_{k-1} L^{k-1}$ characterizes the aggregation scheme. For example, $\omega(L)=1+L+\ldots+$ $L^{k-1}$ in the case of flow variables and $\omega(L)=1$ for stock variables. ${ }^{1}$

Let us also group the first $N$ and $N k$ elements of $y$ and $x$ in the $N \times 1$ and $N k \times 1$ vectors $Y$ and $X$, and construct the $N \times N k$ matrix $W$, with

$$
W=\left(\begin{array}{cccc}
\omega_{0}, \omega_{1}, \ldots \omega_{k-1} & 0,0, \ldots, 0 & \ldots & 0,0, \ldots, 0 \\
0,0, \ldots, 0 & \omega_{0}, \omega_{1}, \ldots \omega_{k-1} & \ldots & 0,0, \ldots, 0 \\
\ldots & & & \\
0,0, \ldots, 0 & 0,0, \ldots, 0 & & \omega_{0}, \omega_{1}, \ldots \omega_{k-1}
\end{array}\right),
$$

so that $Y=W X$.

We assume that the expected loss due to a mismatch between $X$ and its estimator $\widehat{X}$ corresponds to the mean squared disaggregation error

${ }^{1}$ The following analysis remains valid even if $k$ or the weights in $\omega(L)$ change over time. 
(MSDE), so that the problem that we wish to solve can be formulated as:

$$
\begin{gathered}
\min _{\widehat{X}} \operatorname{tr}\left(E(X-\widehat{X})(X-\widehat{X})^{\prime}\right) \\
\text { s.t. } Y=W X .
\end{gathered}
$$

Under the additional hypothesis that $\widehat{X}$ is a linear function of $Y, \widehat{X}=$ $P Y$, the solution is given in the following Proposition.

Proposition 1. The linear minimum MSDE estimator of $X$ is

$$
\widehat{X}^{*}=P^{*} Y=V_{X} W^{\prime} V_{Y}^{-1} Y,
$$

where $V_{X}$ and $V_{Y}$ are the variance covariance matrices of $X$ and $Y$. Moreover, $\widehat{X}^{*}$ is unbiased, satisfies $Y=W \widehat{X}^{*}$, and it is $E\left(X-\widehat{X}^{*}\right)(X-$ $\left.\widehat{X}^{*}\right)^{\prime}=V_{X}-V_{X} W^{\prime} V_{Y}^{-1} W V_{X}$.

Proof Let us consider a generic linear estimator $\widehat{X}=P Y=P W X$. The objective function can then be written as

$$
\operatorname{tr}\left(E(I-P W) X X^{\prime}(I-P W)^{\prime}\right)=\operatorname{tr}\left((I-P W) V_{X}(I-P W)^{\prime}\right) .
$$

For it to be minimised it is necessary that $P^{*}$ satisfies the first order conditions

$$
-V_{X} W^{\prime}+P^{*} W V_{X} W^{\prime}=0 .
$$

This is also sufficient because the second order conditions are also satisfied, given that $W V_{X} W^{\prime}$ is a positive definite matrix. Furthermore, from (2) it follows that $W V_{X} W^{\prime}=V_{Y}$. Hence, the linear minimum MSDE estimator is

$$
\widehat{X}^{*}=P^{*} Y=V_{X} W^{\prime} V_{Y}^{-1} Y
$$

Moreover,

$$
E\left(\widehat{X}^{*}\right)=P^{*} E(Y)=P^{*} W E(X)=0, \text { and } W \widehat{X}^{*}=W V_{X} W^{\prime} V_{Y}^{-1} Y=Y \text {. }
$$


Finally,

$$
\begin{gathered}
E\left(X-\widehat{X}^{*}\right)\left(X-\widehat{X}^{*}\right)^{\prime}=E\left(X-P^{*} W X\right)\left(X-P^{*} W X\right)^{\prime}= \\
E\left(\left(I-P^{*} W\right) X X^{\prime}\left(I-P^{*} W\right)^{\prime}\right)=\left(I-P^{*} W\right) V_{X}\left(I-P^{*} W\right)^{\prime}= \\
\left(\left(I-P^{*} W\right) V_{X}\right)^{\prime}-\left(\left(I-P^{*} W\right) V_{X} W^{\prime} P^{*^{\prime}}\right)^{\prime}= \\
\left(V_{X}-V_{X} W^{\prime} V_{Y}^{-1} W V_{X}\right)^{\prime}-0= \\
V_{X}-V_{X} W^{\prime} V_{Y}^{-1} W V_{X} . \mathbf{}
\end{gathered}
$$

$\widehat{X}^{*}$ can be interpreted as the projection of $X$ on the space spanned by $Y$. When there are no disaggregation problems, i.e. $W=I$, the former Proposition implies that it is $\widehat{X}^{*}=X$ and $E\left(X-\widehat{X}^{*}\right)\left(X-\widehat{X}^{*}\right)^{\prime}=0$.

Notice that we have made no hypotheses at all about the stationarity of $x$ or the functional form of its generating mechanism, except for the existence of the second order moments $V_{X}$. Actually, we have also required $x$ to have zero mean, but this assumption can be simply relaxed by de-meaning $x$ and $y$, and substituting them with the resulting processes. The non zero mean of $x$ can then be simply recovered from that of $y .^{2}$

Instead, if we assume that $x$ is a weakly stationary Gaussian process, $\widehat{X}^{*}$ is the minimum MSDE estimator of $X$, because it coincides with $E(X \mid Y)$. In this case, $\widehat{X}^{*}$ also coincides with the solution of the problem

$$
\begin{aligned}
& \min _{X} X^{\prime} V_{X}^{-1} X \\
& \text { s.t. } Y=W X .
\end{aligned}
$$

Such a solution was provided for this general formulation of the problem but with $\omega(L)=1+L+\ldots+L^{k-1}$ by Stram and Wei (1986), and for particular values of $V_{X}$ by Boot et al. (1967) and Cohen et al. (1971). It can be simply shown that the choice of $\omega(L)$ does not alter the form of the solution.

\footnotetext{
${ }^{2}$ When $x$ has a time varying mean, an identification problem can arise. Actually, from $m_{y}=E(Y)=W E(X)=W m_{x}$, if all the elements of $m_{x}$ are equal (or at least $k$ by $k$ equal), they can be uniquely recovered from those of $m_{y}$. Otherwise, an infinite number of $m_{x}$ are compatible with $m_{y}$, as can be easily derived if we interpret the condition $m_{y}=W m_{x}$ as a set of $N$ equations in $N k$ unknowns.
} 
If instead the hypothesis that $x$ is an ARMA process is maintained, $\widehat{X}^{*}$ coincides with the pure ARMA based minimum MSDE estimator in Guerrero (1990).

For Proposition 1 to be applicable, it is necessary to assume that $x$ follows a particular process, or at least to specify a $V_{X}$. This hypothesis is often made in the literature, see e.g. the aforementioned studies (with the exception of Guerrero (1990)), or Harvey and Pierse (1984), Kohn and Ansley (1986), Nijman and Palm (1986), and Gomez and Maravall (1995) in the missing observations approach. Yet, it seems rather restrictive because many processes or $V_{X} \mathrm{~s}$ can be compatible with their aggregated counterparts, as we will see in more details in Sections 5 and 6 . Hence, an alternative more satisfactory route can be the specification of a set of potential disaggregated processes, together with a subjective probabilistic statement about their likelihood.

To this end, we indicate with $\mathcal{M}$ the set of processes, $\mathcal{M}=\left\{M_{i}\right.$, $i=1, \ldots, m\}$, and with $p_{i}$ the probability of $M_{i}$, where $\sum_{i=1}^{m} p_{i}=1$. Notice that each process in $\mathcal{M}$ has only to be such that $W V_{X}^{i} W^{\prime}=V_{Y}$. Thus, the problem becomes:

$$
\begin{gathered}
\min _{\widehat{X}} \operatorname{tr}\left(E_{M}\left(E(X-\widehat{X})(X-\widehat{X})^{\prime}\right)\right) \\
\text { s.t. } Y=W X,
\end{gathered}
$$

and the solution is given in the following Proposition.

Proposition 2. The linear minimum expected MSDE estimator of $X$ is

$$
\widehat{X}_{M}^{*}=\bar{V}_{X} W^{\prime} V_{Y}^{-1} Y=\sum_{i=1}^{m} \widehat{X}_{M_{i}}^{*} p_{i},
$$

where $\bar{V}_{X}=\sum_{i=1}^{m} V_{X}^{i} p_{i}$. Furthermore, it is unbiased and satisfies $Y=$ $W \widehat{X}_{M}^{*}$.

Proof Let us consider again a generic linear estimator $\widehat{X}=P Y=$ $P W X$ and write the objective function as

$$
\sum_{i=1}^{m} p_{i} \operatorname{tr}\left((I-P W) V_{X}^{i}(I-P W)^{\prime}\right) .
$$


$P^{*}$ has to satisfy the first order conditions

$$
-\bar{V}_{X} W^{\prime}+P^{*} W \bar{V}_{X} W^{\prime}=0,
$$

while the second order conditions are satisfied because $W \bar{V}_{X} W^{\prime}=$ $\sum_{i=1}^{m} p_{i} W V_{X}^{i} W^{\prime}=\sum_{i=1}^{m} p_{i} V_{Y}=V_{Y}$ is positive definite. Hence,

$$
\widehat{X}_{M}^{*}=P^{*} Y=\bar{V}_{X} W^{\prime} V_{Y}^{-1} Y
$$

is the best linear estimator of $X$ in the MSDE sense.

It is also

$$
E\left(\widehat{X}_{M}^{*}\right)=P^{*} E(Y)=0
$$

and

$$
W \widehat{X}_{M}^{*}=W \bar{V}_{X} W^{\prime} V_{Y}^{-1} Y=\sum_{i=1}^{m} p_{i} W V_{X}^{i} W^{\prime} V_{Y}^{-1} Y=Y .
$$

Hence, $\widehat{X}_{M}^{*}$ is a convex linear combination of all the potential "disaggregators", whose weights depend on the relative probability of their associated disaggregated processes (or variance matrices). A continuous cumulative density function for the processes in $\mathcal{M}, F(M)$, could also be adopted. Under the assumption of existence of $\bar{V}_{X}=\int_{\mathcal{M}} V_{M} d F(M)$, the former formula for $\widehat{X}_{M}^{*}$ is still valid.

The procedure can be easily adapted to deal with the multivariate case. For example, in the bivariate case, we can partition $Y$ and $X$ into $\left(Y_{1}, Y_{2}\right)^{\prime}$ and $\left(X_{1}, X_{2}\right)^{\prime}$, where $Y_{1}, Y_{2}, X_{1}$ and $X_{2}$ are respectively $N_{1} \times 1$, $N_{2} \times 1, N_{1} k \times 1$, and $N_{2} k \times 1$ vectors of elements of the aggregated and disaggregated processes $y=\left(y_{1}, y_{2}\right)^{\prime}, x=\left(x_{1}, x_{2}\right)^{\prime}$, and $N_{1}=N_{2}=$ $N / 2$. Then, the optimal estimators of the disaggregated values are $\widehat{X}^{*}=$ $\left(\widehat{X}_{1}^{*}, \widehat{X}_{2}^{*}\right)^{\prime}$ in $(3)$.

The procedure can be also used to recover disaggregated values from linearly aggregated series by simply redefining the involved variables. For example, $X$ can be interpreted as a vector containing $N$ variables for $k$ agents, while in $Y$ the variables are aggregated over agents.

Finally, joint temporal and linear disaggregation can be easily dealt with in a similar manner. This case is considered by Rossi (1982), whose 
method can be seen as a mixture of those by Denton (1971) and Chow and Lin (1971), which are reviewed in next Section, and in Di Fonzo (1990), who extends Chow and Lin's approach.

\section{Some Extensions}

We now extend the basic results and procedures in the former Section. First, we deal with estimation of the unobservable series at several disaggregation frequencies, and with observable series at different levels of aggregation. Then we consider the case where a preliminary estimate of $X$ is available. Finally, we derive the optimal disaggregator when the objective function is the generalized variance of the disaggregation errors.

\subsection{Multiple temporal frequencies}

For simplicity but without loss of generality, let us assume that probability one is assigned to one process in $\mathcal{M}$, and that neither $X_{1}$ nor $X$ can be observed where, in an obvious notation,

$$
\begin{gathered}
X_{1}=\underset{N k_{1} \times 1}{W_{2}} \underset{N k_{1} \times N k N k \times 1}{X}, \\
\underset{N \times 1}{Y}=\underset{N \times N k_{1} N k_{1} \times 1}{W_{1}}=W_{1} W_{2} X=W X,
\end{gathered}
$$

with $k=k_{1} k_{2}$.

To find the linear minimum MSDE estimators of $X$ and $X_{1}$ we can apply the formula in (3), which yields $\widehat{X}^{*}=V_{X} W^{\prime} V_{Y}^{-1} Y$ and $\widehat{X}_{1}^{*}=$ $V_{X_{1}} W_{1}^{\prime} V_{Y}^{-1} Y$. As an alternative, we can exploit $\widehat{X}^{*}$ or $\widehat{X}_{1}^{*}$ to derive, respectively, the best estimators of $X_{1}$ and $X$. For example, we can estimate monthly and quarterly data from annual data, or we can use the estimated monthly data to derive quarterly data, or vice versa. To this end, we have

$$
\text { Proposition 3. } \widehat{X}_{1}^{*}=W_{2} \widehat{X}^{*} \text { and } \widehat{X}^{*}=V_{X} W^{\prime}\left(W_{1} V_{\widehat{X}_{1}^{*}} W_{1}^{\prime}\right)^{-1} W_{1} \widehat{X}_{1}^{*}
$$


Proof From (3) we know that $\widehat{X}_{1}^{*}=V_{X_{1}} W_{1}^{\prime} V_{Y}^{-1} Y$ and $\widehat{X}^{*}=V_{X} W^{\prime} V_{Y}^{-1} Y$. It follows that

$$
W_{2} \widehat{X}^{*}=W_{2} V_{X} W_{2}^{\prime} W_{1}^{\prime} V_{Y}^{-1} Y=V_{X_{1}} W_{1}^{\prime} V_{Y}^{-1} Y=\widehat{X}_{1}^{*} .
$$

Moreover,

$$
\begin{aligned}
V_{X} W^{\prime}\left(W_{1} V_{\widehat{X}_{i}^{*}} W_{1}^{\prime}\right)^{-1} W_{1} \widehat{X}_{1}^{*} & =V_{X} W^{\prime} V_{Y}^{-1} W_{1} V_{X_{1}} W_{1}^{\prime} V_{Y}^{-1} Y= \\
& =V_{X} W^{\prime} V_{Y}^{-1} Y=\widehat{X}^{*} .
\end{aligned}
$$

Hence, the best linear estimators of the aggregated series can be obtained by aggregating the most disaggregated estimators. Instead, there are no major computational advantages in using the aggregate estimators to determine the disaggregated ones.

A further topic to be considered is the role of observable series at different levels of aggregation. For example, we can assume that both $Y$ and $X_{1}$ are observable, and we have to decide which one to employ in order to estimate $X$.

Proposition 4. Given $\widehat{X}^{* Y}=V_{X} W^{\prime} V_{Y}^{-1} Y$ and $\widehat{X}^{* X_{1}}=V_{X} W_{2}^{\prime} V_{X_{1}}^{-1} X_{1}$, it is $W_{2} \widehat{X}^{* Y} \neq X_{1}$, and $\widehat{X}^{* Y}$ is less efficient than $\widehat{X}^{* X_{1}}$ in the sense that it leads to a larger MSDE.

Proof $\widehat{X}^{* Y}$ and $\widehat{X}^{* X_{1}}$ are obtained by solving the minimization problem in (1) under, respectively, the constraints in (2) and $X_{1}=W_{2} X$. Hence, it is, $W_{2} \widehat{X}^{* Y}=V_{X_{1}} W_{1}^{\prime} V_{Y}^{-1} W_{1} X_{1} \neq X_{1}$. This happens because the constraint $X_{1}=W_{2} X$ is not considered in the first optimization problem, while (2) is also implicitly imposed in the problem which leads to $\widehat{X}^{* X_{1}}$.

Then, we have:

$$
\begin{gathered}
E\left(X-\widehat{X}^{* Y}\right)\left(X-\widehat{X}^{* Y}\right)^{\prime}=E\left(\left(X-\widehat{X}^{* X_{1}}\right)-\left(\widehat{X}^{* X_{1}}-\widehat{X}^{* Y}\right)\right)\left(\left(X-\widehat{X}^{* X_{1}}\right)+\right. \\
\left.-\left(\widehat{X}^{* X_{1}}-\widehat{X}^{* Y}\right)\right)^{\prime}=E\left(X-\widehat{X}^{* X_{1}}\right)\left(X-\widehat{X}^{* X_{1}}\right)^{\prime}+ \\
+E\left(X-\widehat{X}^{* X_{1}}\right)\left(\widehat{X}^{* X_{1}}-\widehat{X}^{* Y}\right)^{\prime}+ \\
E\left(\widehat{X}^{* X_{1}}-\widehat{X}^{* Y}\right)\left(X-\widehat{X}^{* X_{1}}\right)^{\prime}+E\left(\widehat{X}^{* X_{1}}-\widehat{X}^{* Y}\right)\left(\widehat{X}^{* X_{1}}-\widehat{X}^{* Y}\right)^{\prime}
\end{gathered}
$$


But,

$$
\begin{gathered}
E\left(X-\widehat{X}^{* X_{1}}\right)\left(\widehat{X}^{* X_{1}}-\widehat{X}^{* Y}\right)^{\prime}= \\
=\left(I-V_{X} W_{2}^{\prime} V_{X_{1}}^{-1} W_{2}\right) V_{X} W_{2}^{\prime}\left(V_{X_{1}}^{-1}-W_{1}^{\prime} V_{Y}^{-1} W_{1}\right) W_{2} V_{X}= \\
V_{X} W_{2}^{\prime}\left(V_{X_{1}}^{-1}-W_{1}^{\prime} V_{Y}^{-1} W_{1}\right) W_{2} V_{X}+ \\
-V_{X} W_{2}^{\prime} V_{X_{1}}^{-1} V_{X_{1}}\left(V_{X_{1}}^{-1}-W_{1}^{\prime} V_{Y}^{-1} W_{1}\right) W_{2} V_{X}=0,
\end{gathered}
$$

and similarly it can be shown that $E\left(\widehat{X}^{* X_{1}}-\widehat{X}^{* Y}\right)\left(X-\widehat{X}^{* X_{1}}\right)^{\prime}=0$.

Hence, it is:

$$
\begin{aligned}
& E\left(X-\widehat{X}^{* Y}\right)\left(X-\widehat{X}^{* Y}\right)^{\prime}-E\left(X-\widehat{X}^{* X_{1}}\right)\left(X-\widehat{X}^{* X_{1}}\right)^{\prime} \\
= & E\left(\widehat{X}^{* X_{1}}-\widehat{X}^{* Y}\right)\left(\widehat{X}^{* X_{1}}-\widehat{X}^{* Y}\right)^{\prime},
\end{aligned}
$$

so that the term on the left hand side is positive definite. This also implies that

$$
\operatorname{tr}\left(E\left(X-\widehat{X}^{* Y}\right)\left(X-\widehat{X}^{* Y}\right)^{\prime}\right)-\operatorname{tr}\left(E\left(X-\widehat{X}^{* X_{1}}\right)\left(X-\widehat{X}^{* X_{1}}\right)^{\prime}\right)>0,
$$

i.e., $\widehat{X}^{* Y}$ leads to a larger MSDE than $\widehat{X}^{* X_{1}}$.

This result suggests the adoption of the most disaggregated available series for further disaggregation.

\subsection{Updating of preliminary estimates}

We now wish to modify our procedure to deal with the case where a preliminary estimate of $X, Z$, is available. As it is often assumed in what we have defined the third disaggregation method in the Introduction, we hypothesise that $Z$ does not satisfy the constraints in (2), namely $Y \neq W Z$. Hence, the problem that we have to solve can be stated as

$$
\begin{gathered}
\min _{\widehat{X}} \operatorname{tr}\left(E(X-\widehat{X})(X-\widehat{X})^{\prime} \mid Z\right) \\
\text { s.t. } Y=W X .
\end{gathered}
$$

The solution is given in the following Proposition. 
Proposition 5. The linear minimum MSDE estimator of $X$ is

$$
\widehat{X}^{*}=Z+V_{X} W^{\prime} V_{Y}^{-1}(Y-W Z) .
$$

Proof If we define $S=X-Z, \widehat{S}=\widehat{X}-Z$, and $T=Y-W Z$, the problem in (8) can be reformulated as

$$
\min _{\widehat{S}} \operatorname{tr}\left(E(S-\widehat{S})(S-\widehat{S})^{\prime} \mid Z\right)
$$

$$
\text { s.t. } T=W S \text {. }
$$

It is also $V(S \mid Z)=V(X)$ and $V(T \mid Z)=V(Y)$. Thus, from Proposition 1 , it follows that $\widehat{S}^{*}=V_{S} W^{\prime} V_{T}^{-1} T$ and

$$
\widehat{X}^{*}=\widehat{S}^{*}+Z=Z+V_{X} W^{\prime} V_{Y}^{-1}(Y-W Z) \text {. }
$$

Therefore, $\widehat{X}^{*}$ in (3) coincides with that in (9) when $Z=0$.

Denton (1971) proposed to

$$
\begin{gathered}
\min _{X}(X-Z)^{\prime} D(X-Z) \\
\text { s.t. } Y=W X,
\end{gathered}
$$

where $D$ is an $N k \times N k$ deterministic matrix which depends on a penalty function. The optimal estimator is $\widetilde{X}^{*}=Z+D^{-1} W^{\prime}\left(W D^{-1} W^{\prime}\right)^{-1}(Y-$ $W Z$ ). It follows that $\widehat{X}^{*}$ in (9) is also a solution for this problem when $D=V_{X}^{-1}$.

Finally, let us consider a $g$ dimensional disaggregated observable stochastic process $s=\left\{s_{t}\right\}_{t=1}^{\infty}$, group its first $N k$ realizations into the $N k \times g$ matrix $S$, and assume that there exists a static stochastic linear relationship between $x_{t}$ and $s_{t}$. If we call $\hat{b}$ the $G L S$ estimator in a regression of $y_{t}$ on $W s_{t}$, then a preliminary estimate of $X$ is $Z=W S \widehat{b}$. For such a choice of $Z,(9)$ is equal to the best linear unbiased estimator of $X$ in Chow and Lin (1971). 


\subsection{A different objective function}

Clements and Hendry (1993) criticize the adoption of mean squared forecast error as a criterion for the evaluation of the forecasting performance of a model, on the grounds that it can be not invariant to isomorphic transformations and does not take into account cross correlation among forecast errors. Their suggestion is to adopt the generalized variance as a criterion, which solves both these problems. Actually, whether these are problems or not can be disputed upon, because an investigator might be interested in a particular specification of the model and willing to ignore cross correlation among forecast errors. However, it seems interesting to study what happens in our framework if we substitute the mean square disaggregation error with the generalized variance, $\operatorname{det}\left(E(X-\widehat{X})(X-\widehat{X})^{\prime}\right)$, as the objective function. Thus, we wish to solve the problem:

$$
\begin{gathered}
\min _{\widehat{X}} \operatorname{det}\left(E(X-\widehat{X})(X-\widehat{X})^{\prime}\right) \\
\text { s.t. } \quad Y=W X .
\end{gathered}
$$

If we limit the potential solutions to the set $\widehat{X}=P Y$, we have $\operatorname{det}\left(E(X-\widehat{X})(X-\widehat{X})^{\prime}\right)=\operatorname{det}\left(E(X-P W X)(X-P W X)^{\prime}\right)=$ $\operatorname{det}\left((I-P W) V_{X}(I-P W)^{\prime}\right)=\operatorname{det}(I-P W) \operatorname{det}\left(V_{X}\right) \operatorname{det}(I-P W)^{\prime}$.

Therefore, we can reformulate the problem in (11) as:

$$
\min _{P}|\operatorname{det}(I-P W)|,
$$

where | | indicates the absolute value.

If we define $\tilde{\omega}=\omega_{0}+\omega_{1}+\ldots+\omega_{k-1}$, and

$$
\begin{aligned}
\underset{1 \times k}{p^{\prime}} & =(1 / \widetilde{\omega}, 1 / \widetilde{\omega}, \ldots, 1 / \widetilde{\omega}), \\
\underset{N k \times N}{P^{*}} & =\left(\begin{array}{cccc}
p & 0 & \ldots & 0 \\
0 & p & \ldots & 0 \\
\ldots & & & \\
0 & 0 & \ldots & p
\end{array}\right)
\end{aligned}
$$


then,

Proposition 6. $P^{*}$ solves the problem in (12).

Proof We have:

$$
\begin{aligned}
I \underset{N k \times N k}{P} W & =\left(\begin{array}{cccc}
Q & 0 & \ldots & 0 \\
0 & Q & \ldots & 0 \\
\ldots & & & \\
0 & 0 & \ldots & Q
\end{array}\right), \\
\underset{k \times k}{Q} & =\left(\begin{array}{cccc}
1-\omega_{0} / \tilde{\omega} & -\omega_{1} / \tilde{\omega} & \ldots & -\omega_{k-1} / \tilde{\omega} \\
-\omega_{0} / \tilde{\omega} & 1-\omega_{1} / \tilde{\omega} & \ldots & -\omega_{k-1} / \tilde{\omega} \\
\ldots & & & \\
-\omega_{0} / \tilde{\omega} & -\omega_{1} / \tilde{\omega} & \ldots & 1-\omega_{k-1} / \tilde{\omega}
\end{array}\right) .
\end{aligned}
$$

The first column in $Q$ is equal to the sum of the other $k-1$ columns, with the opposite sign. Hence, $\operatorname{det}(Q)=0$, and $|\operatorname{det}(I-P W)|=0$.

This leads to, and provides a justification for, very naive disaggregation methods. For example, in the case of a monthly process transformed into a quarterly process by average sampling, an optimal monthly estimate is equal to one third of the corresponding quarterly figure. It is exactly equal to the quarterly figure in the case of point-in-time sampling.

Yet, these values are quite unrealistical, and the fact that the "disaggregator" is completely independent of the original process $x$ is also hard to accept, even if it can be a useful property when no disaggregate information is available. Hence, in the remainder of the paper we stick to the original formulation of the objective function, the MSDE, and to the related best disaggregator.

\section{Missing observations, outliers, and fore- casting}

The procedure in Section 2, possibly extended as in Section 3, can be also easily adapted to provide linear minimum MSDE estimators of missing 
observations. Actually, this only requires a proper choice of $W$. For example, if the $n_{i}^{\text {th }}$ observations are missing, $i=1, \ldots, m, W$ has to be equal to an identity matrix whose $n_{i}^{\text {th }}$ rows have been deleted.

We now explicitly consider the formula of the optimal estimator when the first observation is missing, because it is useful to highlight the relationship with former results.

Proposition 7. If the first observation is missing, the optimal estimator in (3) becomes:

$$
\widehat{X}^{*}=\left(\begin{array}{c}
p \\
1 \times N k-1 \\
I \\
I k-1 \times N k-1
\end{array}\right) Y,
$$

where $p^{*}=v_{1 .} V_{Y}^{-1}, v_{1 .}=\left(v_{12}, v_{13}, \ldots, v_{1 N k}\right), v_{1 j}=\operatorname{cov}\left(x_{1}, x_{j}\right)$. Moreover, the variance of the estimator of the missing observation is $v_{11}-v_{1} . V_{Y}^{-1} v_{1}^{\prime}$.

Proof In this case it is

$$
\underset{N k \times N k-1}{V_{X} W^{\prime}}=\left(\begin{array}{cccc}
v_{12} & v_{13} & \ldots & v_{1 N k} \\
v_{22} & v_{23} & \ldots & v_{2 N k} \\
\ldots & & & \\
v_{N k 2} & v_{N k 3} & \ldots & v_{N k N k}
\end{array}\right),
$$

and

$$
V_{Y}=\underset{N k-1 \times N k-1}{W V_{X} W^{\prime}}=\left(\begin{array}{cccc}
v_{22} & v_{23} & \ldots & v_{2 N k} \\
v_{32} & v_{33} & \ldots & v_{3 N k} \\
\ldots & & & \\
v_{N k 2} & v_{N k 3} & \ldots & v_{N k N k}
\end{array}\right)
$$

Given that $P^{*}=V_{X} W^{\prime} V_{Y}^{-1}$, it must be $P^{*} V_{Y}=V_{X} W^{\prime}$. Let us partition $P^{*}, P^{*} V_{Y}$ and $V_{X} W^{\prime}$ as

$$
P^{*}=\left(\begin{array}{c}
p \\
1 \times N k-1 \\
P \\
P k-1 \times N k-1
\end{array}\right), \quad P^{*} V_{Y}=\left(\begin{array}{l}
p V_{Y} \\
P V_{Y}
\end{array}\right), \quad V_{X} W^{\prime}=\left(\begin{array}{l}
v_{1} \\
V_{Y}
\end{array}\right) .
$$

It follows that it must be $P=I$ and $p=v_{1} \cdot V_{Y}^{-1}$. This also implies that the variance of the estimator of the missing observation is $v_{11}-$ $v_{1 .} V_{Y}^{-1} v_{.1}^{\prime}$. 
Hence, the best estimators of the available observations are the ob̈servations themselves, as one could have expected, while that of the missing observation is a linear combination of the available ones. A more familiar interpretation of the weights is possible. Actually,

Proposition 8. The $i^{\text {th }}$ element of $p^{*}$ is equal to the negative of the $i^{\text {th }}$ lag of the inverse autocorrelation function, $\rho_{i}$, of $x, i=1, \ldots, N k-1$.

Proof Kato (1984) showed that the inverse autocorrelation function at lag $j$ is equal to the negative of the partial correlation between $x_{t}$ and $x_{t-j}$. From Brockwell and Davis (1991, p. 102), the partial correlations between $x_{t}$ and $x_{t-j}, j=1, \ldots, N k-1$, can be written, in our notation, as $v_{1} . V_{Y}^{-1}$.

The inverse autocorrelation function is the basic tool in the literature on the estimation of missing observations. In particular, under the additional hypothesis that $x$ is a doubly infinite process, it is well known that the minimum MSDE estimator of the missing observation $x_{i}$ is:

$$
\widehat{x}_{i}^{*}=-\sum_{j=1}^{\infty} \rho_{j}\left(x_{i-j}+x_{i+j}\right)
$$

see, e.g., Whittle (1963). (14) can be seen as the limit of

$$
\widehat{x}_{i}^{*}=-\sum_{j=1}^{(N k-1) / 2} \rho_{j}\left(x_{i-j}+x_{i+j}\right),
$$

when $N$ tends to infinity, where (15) is our optimal estimator for the mid-sample observation.

We now hypothesize that the series has additive outliers, $w_{i}$, in periods $i=n_{1}, \ldots, n_{m}$, so that the observed values are $y_{i}=x_{i}+w_{i}$. The optimal linear estimators of the outliers, $\widehat{w}_{i}^{*}$, can be constructed by subtracting from $y_{i}$ the optimal estimators of $x_{i}$, when $x_{i}$ are treated as missing observations, i.e.,

$$
\widehat{w}_{i}^{*}=y_{i}-\widehat{x}_{i}^{*} .
$$

Their variance covariance matrix is equal to that of the estimators of the missing observations. This approach was followed, e.g., in Peña and 
Maravall (1991) just to relate the estimation of outliers and missing observations.

As an example, if there is one outlier in the middle of the sample, its best linear estimator according to our procedure is:

$$
\widehat{w}_{i}^{*}=y_{i}+\sum_{j=1}^{(N k-1) / 2} \rho_{j}\left(x_{i-j}+x_{i+j}\right) \text {. }
$$

The limit of this expression when $N$ diverges coincides with the formula for the optimal estimator of an additive outlier in Chang et al. (1988).

Finally, notice that forecasting can be considered as a problem of estimation of a set of missing observations at the end of the sample. Hence, linear minimum MSFE forecasts, and their associated standard errors, can be also easily obtained by means of a proper choice of $W$.

\section{Identification of $\mathbf{V}_{x}$}

So far we have hypothesised that $V_{x}$ or the form of the original process are known. Instead, we now investigate whether and how available aggregate information can be used to infer them. In the next Section we examine the links between the aggregated and disaggregated generating processes, while in this Section we analyse in greater details the relationship between $V_{x}$ and $V_{y}$. In particular, we have noticed that

$$
W V_{X} W^{\prime}=V_{Y}
$$

because of the deterministic relationship $Y=W X$. We now consider under what conditions $V_{X}$ can be uniquely determined given $V_{Y}$ and $W$.

It is useful to partition $X$ into $X=\left(X_{1}, X_{2}, \ldots, X_{N}\right)^{\prime}$, where $X_{1}=$ $\left(x_{1}, x_{2}, \ldots, x_{k}\right)^{\prime}, X_{2}=\left(x_{k+1}, x_{k+2}, \ldots, x_{2 k}\right)^{\prime}$, and so on. $V_{X}$ can then be partitioned into the $N^{2} k \times k$ matrices $C_{i, j}=\operatorname{cov}\left(X_{i}, X_{j}\right), i, j=1, \ldots, N$. We also define $w=\left(\omega_{0}, \ldots, \omega_{k-1}\right)$ and indicate with $v_{i, j}^{X}, v_{i, j}^{Y}$, the elements in the $\mathrm{i}^{\text {th }}$ row and $\mathrm{j}^{\text {th }}$ column of $V_{X}$ and $V_{Y}$. 
It follows that (18) can be rewritten as

$$
w C_{i, j} w^{\prime}=v_{i, j}^{Y}, \quad i, j=1, \ldots N .
$$

Thus, $v_{i, j}^{Y}$ is a linear combination of the elements in $C_{i, j}$, whose weights depend on the aggregation scheme.

In (19) there are $N^{2}$ equations in $N^{2} k^{2}$ unknowns (the elements of $V_{X}$ ), which can be reduced to $N(N+1) / 2$ equations in $N k(N k+1) / 2$ unknowns because of symmetry of $V_{X}$ and $V_{Y}$. Hence, in general, there are $d=\left(N^{2}\left(k^{2}-1\right)+N(k-1)\right) / 2$ degrees of freedom in the determination of the elements of $V_{X}$ given those of $V_{Y}$. Thus, a necessary condition to exactly identify $V_{X}$, is the imposition of $d$ a priori restrictions on its elements, which can be, e.g., in the form of exclusion or linear restrictions.

In the case of weak stationarity of $X$ the number of degrees of freedom is much lower but still high. Actually, (19) can be reduced to

$$
w C_{1, j} w^{\prime}=v_{1, j}^{Y}, \quad j=1, \ldots N,
$$

because knowledge of $C_{1,1}, \ldots, C_{1, N}$ is sufficient to recover $V_{X}$. (20) can be also rewritten in the linear system formulation

$$
A v^{X}=v^{Y}
$$

where $v^{X}=\left(v_{1,1}^{X}, v_{1,2}^{X}, \ldots, v_{1, N K}^{X}\right)^{\prime}, v^{Y}=\left(v_{1,1}^{Y}, v_{1,2}^{Y}, \ldots, v_{1, N}^{Y}\right)^{\prime}$,

$$
\underset{N \times N K}{A}=\left(\begin{array}{cccccccccccc}
a_{k} & 2 a_{k-1} & 2 a_{k-2} & \ldots & 2 a_{1} & 0 & 0 & & & & 0 \\
0 & a_{1} & a_{2} & \ldots & a_{k-1} & a_{k} & a_{k-1} & \ldots & 0 & \ldots & 0 \\
0 & 0 & a_{1} & \ldots & a_{k-2} & a_{k-1} & a_{k} & \ldots & a_{1} & \ldots & 0 \\
\ldots & & & & & & & & & & \\
0 & 0 & 0 & \ldots & 0 & 0 & 0 & \ldots & 0 & \ldots & a_{1}
\end{array}\right)
$$

and $a_{j}=\sum_{i=0}^{j-1} \omega_{i} \omega_{i+k-j}, j=1, \ldots, k$. A similar expression was derived by Wei and Stram (1990) for the case $w=(1,1, \ldots, 1)$.

In (21) there are $N$ equations in $N k$ unknowns. Therefore, the imposition of $N k-N$ a priori restrictions on $v^{X}$ is a necessary condition for its exact identification. A useful example of the non sufficiency of such 
a condition is that of point-in-time sampling, where $w=(1,0, \ldots, 0)$. In this case, it is $v_{1,1+k j}^{X}=v_{1,1+j}^{Y}, j=0, \ldots, N-1$, and the other elements of $v^{X}$ are completely unrestricted. If they are set equal to fixed numbers $v^{X}$ is exactly identified, but if some of the $v_{1,1+k j}^{X}$ are set equal to arbitrary fixed numbers, the system in (21) cannot be solved.

Instead, we have,

Proposition 9. If $N k-N$ elements of $v^{X}$ are set equal to fixed numbers, the corresponding columns of $A$ are deleted in order to obtain the $N \times N$ matrix $\widetilde{A}$, and the unrestricted elements of $v^{X}$ are grouped in the $N \times 1$ vector $\tilde{v}^{X}$, sufficient condition for $v^{X}$ to be exactly identified is that $|\tilde{A}| \neq 0$, which implies that it is $\widetilde{v}^{X}=\tilde{A}^{-1} v^{Y}$.

Proof The proof is immediate.

When a small subset of observations are missing, it is in general possible to recover $v^{X}$ from $v^{Y}$. A relevant exception is when the first or last $k$ observations are missing. In this case, it is not possible to recover the $k$ highest lags of $v^{X}$.

In practice, $v^{Y}$ is also not known and has to be estimated from a set of $N$ realizations. It follows that the estimates of the higher order covariances of $Y$ can be rather inaccurate, and the inaccuracy is transmitted to those of $X$.

To summarize, given the relationship between $V^{X}$ and $V^{Y}$ in (19) or (21), an infinite number of $V^{X} \mathrm{~S}$ is compatible with $V^{Y}$ in the disaggregation case. When one or more $V^{X}$ are identified by means of a priori restrictions, the methods in the former Sections can be used to obtain the best linear estimator of $X$. Yet, it can be easier to formulate a priori restrictions on disaggregated processes than directly on the elements of $V^{X}$. Thus, we move to studying the relationship between the aggregated and disaggregated generating processes. 


\section{Identification of the disaggregated process}

We now consider the links between the aggregated and disaggregated process, under the assumption that the latter belongs to the ARIMA class. This is a convenient assumption because ARIMA processes seem to adequately statistically characterize many time series, and they can provide accurate approximations also for the $V_{x}$ s which would be implied by a nonlinear process. There is also a major computational advantage in deriving $\widehat{X}^{*}$ under the hypothesis that $x$ is an ARIMA process because the order of the matrices $V_{X}$ and $V_{Y}$ can be reduced to that of the MA component of the $x$ and $y$ processes, as shown by Anderson (1974). Moreover, the modification in the structure of a non linear process due to temporal aggregation is difficult to handle, being model specific.

In the first subsection we briefly recall some results on temporal aggregation of ARIMA processes, which provides a useful background for the following discussion. In the second subsection we identify the set of ARIMA processes which is compatible with a given aggregate ARIMA process for $y$. The third subsection presents an example which illustrates the theoretical findings.

\subsection{Temporal Aggregation of an ARIMA process}

Let us assume that the elements of $x$ satisfy the stochastic difference equations

$$
g(L) x_{t}=f(L) e_{t}
$$

where $g(L)$ and $f(L)$ are polynomials of degree $g$ and $f$ in the lag operator, $L$, the roots of $f(l)=0$ are assumed to lie outside the unit circle, and $e_{t}$ is white noise (WN) with $V\left(e_{t}\right)=\sigma_{e}$. Given this specification, we are interested in determining the generating mechanism of $y$.

Such an issue has been dealt with by many authors in the past. The method that we review in this subsection is that proposed by Brewer (1973), refined by Wei (1981) and Weiss (1984), and further extended in Marcellino (1996) to deal with general aggregation schemes, multivariate 
processes, and determine the coefficients of the MA component. It requires an introduction of a polynomial in the lag operator, $b(L)$, whose degree in $L$ is at most equal to $g k-g$ and which is such that the product $h(L)=b(L) g(L)$ only contains powers of $L^{k}$. It can be shown that such a polynomial always exists, and its coefficients depend on those of $g(L)$, see the above references for details.

In order to determine the AR component of the aggregate ARIMA process, we then multiply both sides of $(22)$ by $\omega(L)$ and $b(L)$ to get

$$
h\left(L^{k}\right) \omega(L) x_{t}=f(L) b(L) \omega(L) e_{t} .
$$

The left hand side of (23) can be also written as

$$
h(Z) y_{\tau}
$$

where $Z=L^{k}$ is the lag operator at the aggregate temporal frequency, i.e., $Z y_{\tau}=y_{\tau-1}$. Thus, the order of the aggregate AR component, $h$, is at most equal to $g$.

Notice that $h(Z)$ can be decomposed into

$$
\prod_{i=1}^{h} \prod_{j=1}^{k}\left(1-\frac{1}{h_{i j}} L\right)
$$

and at least one $h_{i j}$ for each $i$ has to be such that $g\left(h_{i j}\right)=0$.

It can then be shown that the order of the aggregate MA component, $q$, coincides with the highest multiple of $k$ non zero lag in the autocovariance function of $f(L) b(L) \omega(L) e_{t}$. Its coefficients have to be such that the implied autocovariances coincide with those of $f(L) b(L) \omega(L) e_{t}$ evaluated at all multiples of $k$.

In summary, $y$ evolves according to:

$$
h(Z) y_{\tau}=q(Z) u_{\tau}
$$

where $u_{\tau}$ is white noise and $V\left(u_{\tau}\right)=\sigma_{u}$. 


\subsection{Identification of the disaggregated ARIMA process}

Having set up a framework which relates the disaggregated and aggregated ARIMA processes, we now have to determine what and how many ARIMA processes are compatible with an aggregated one. To start with, assuming that $y$ follows the model in (25), we try and identify the $g(L)$ polynomials which can have generated $h(Z)$. This requires to analyse all the possible decompositions of $h\left(L^{k}\right)$ into $b(L) g(L)$.

We have said that at least one $h_{i j}$ for each $i$ in (24) has to be such that $g\left(h_{i j}\right)=0$. The other $k-1 h_{i j} \mathrm{~s}$ can instead solve either $b\left(h_{i j}\right)=0$ or also $g\left(h_{i j}\right)=0$. Thus, for each $i$, there are $2^{k}-1$ possible "distributions" of the $h_{i j} \mathrm{~s}$ as roots of $b(L)$ and $g(L)$. Hence, we obtain a total of $\left(2^{k}-1\right)^{h}$ potential disaggregated AR components, which can be written as

$$
\prod_{m}\left(1-\frac{1}{h_{m}} L\right),
$$

where the $h_{m} \mathrm{~s}$ are the $h_{i j} \mathrm{~s}$ which are considered as roots of $g(l)=0$. The possible degree of $g(L)$ ranges from $h$ to $h k$.

Let us now consider the disaggregated MA component. Its coefficients have to be such that the autocovariances at lags multiple of $k$ are equal to those of the aggregated MA component, whose first $q$ values are different from zero. Given a decomposition of $h(Z)$ into $b(L)$ and $g(L)$, this condition is usually not sufficient to exactly identify one disaggregated MA component but an infinite number of them is admissible, which mirrors the existence of an infinite number of $V_{X} \mathrm{~s}$ which are compatible with $V_{Y}$. As in that situation, it is necessary to resort to a priori restrictions to obtain exact identification.

A simple but rather stringent condition for exact identification of the disaggregate process is:

Proposition 10. All the roots of $g(l)=0$ are distinct and positive, or distinct and possibly negative if $k$ is even, and $f \leq g$, or $f \leq g-1$ in the case of point-in-time sampling.

Proof If $g(l)=0$ has distinct and positive roots, or distinct and possibly negative roots if $k$ is even, then they coincide with those of $h(z)=0$ raised 
to power of $1 / k$, and this exactly identifies the AR component. Moreover, in this case, the aggregated MA component is of order $g$, or $g-1$ for point-in-time sampling. Hence, the at most $g$, or $g-1$, coefficients of the (invertible) disaggregate MA component have to satisfy $g$, or $g-1$, conditions, and this is possible only if they coincide with those of $f(L)$.

Wei and Stram (1990) discuss more general sufficient a priori conditions for one disaggregate model to be identifiable from an aggregate one. As an alternative, many models can be selected on the basis of subjective judgments and the formula in (6) used to determine $\widehat{X}_{M}^{*}$.

Finally, it can be worthwhile pointing out that the procedure can be applied even if the variables are integrated of order $d$. Actually, in this case $X=\left(x_{1}, \ldots, x_{N k}\right)^{\prime}$ and $Y=W X$ are non stationary, but they still have finite second order moments. As an alternative, after having determined the best estimators for the stationary $d^{\text {th }}$ differences of the variables, $\widehat{d X}^{*}$, a method which has been proposed by Stram and Wei (1986) can be adopted to recover the disaggregated values in levels. ${ }^{3}$

Actually, they show that it is

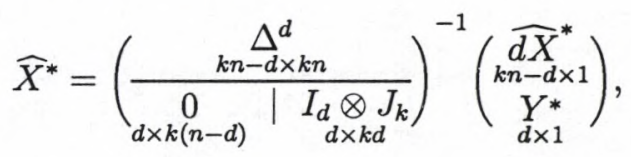

where $I_{d}$ is a $d \times d$ identity matrix, $J_{k}$ is a $1 \times k$ vector of ones, $\otimes$ denotes the Kronecker product, $Y^{*}$ is a $d \times 1$ vector which contains the last $d$ elements in $Y$, and

$$
\Delta^{d}=\left(\begin{array}{ccccccccc}
d_{0} & d_{1} & \ldots & d_{d} & 0 & \ldots & & & 0 \\
0 & d_{0} & d_{1} & \ldots & d_{d} & 0 & \ldots & & 0 \\
\ldots & & & & & & & & \\
0 & \ldots & & 0 & \ldots & & & d_{d-1} & d_{d}
\end{array}\right),
$$

where $d_{i}$ is the coefficient of $L^{i}$ in $(L-1)^{d}$.

\footnotetext{
${ }^{3}$ We recall that under the assumption of joint normality, our optimal estimator coincides with that in Stram and Wei (1986), even if they are derived by solving different optimization problems, see Section 2.
} 


\subsection{An example}

We now discuss an example in order to illustrate the identification problem which has been highlighted in the former subsection. A simple ARMA $(1,1)$ disaggregated process whose realizations are subject to average sampling with $k=2$ is enough to this end.

Hence, we assume that

$$
\left(1-g_{1} L\right) x_{t}=\left(1+f_{1} L\right) e_{t}, \quad e_{t} \sim W N(0,1),
$$

and it can be simply verified, by applying the procedure in subsection 6.1, that the aggregate model is still $\operatorname{ARMA}(1,1)$. In particular, it is

$$
\left(1-h_{1} Z\right) y_{\tau}=\left(1+q_{1} Z\right) u_{\tau}, \quad u_{\tau} \sim W N\left(0, \sigma_{u}\right),
$$

with $h_{1}=g_{1}^{2}, q_{1}=c / 2-\sqrt{c^{2} / 4-1}, c=\gamma_{0} / \gamma_{2}, \gamma_{0}=1+\left(1+g_{1}+\right.$ $\left.f_{1}\right)^{2}+\left(g_{1}+f_{1}+g_{1} f_{1}\right)^{2}+g_{1}^{2} f_{1}^{2}, \gamma_{2}=\left(1+g_{1}+f_{1}\right) g_{1} f_{1}+\left(g_{1}+f_{1}+g_{1} f_{1}\right)$, $\sigma_{u}=\gamma_{2} / f_{1}, \gamma_{0}$ and $\gamma_{2}$ are the variance and autocovariance at lag 2 of $\left(1+g_{1} L\right)(1+L)\left(1+f_{1} L\right) e_{t}$, and we hypothesise $c>6$.

We now have to consider what disaggregate ARMA models are compatible with that in (29). According to the derivation in subsection $6.2,\left(2^{k}-1\right)^{h}=3$ potential disaggregated AR components could have generated that in (29). They are:

a) $\left(1-g_{1}^{2} L^{2}\right)$ which corresponds to $b(L)=1$;

b) $\left(1-g_{1} L\right)$ which corresponds to $b(L)=\left(1+g_{1} L\right)$;

c) $\left(1+g_{1} L\right)$ which corresponds to $b(L)=\left(1-g_{1} L\right)$.

For each of them, we have to identify the set of potential disaggregate MA components. In the case of (30a) the order of the MA component has to be larger than or equal to one. If it is equal to one, i.e.,

$$
\text { a1) }\left(1-g_{1}^{2} L^{2}\right) x_{t}=\left(1+f_{1}^{a 1} L\right) e_{t}^{a 1}, \quad e_{t}^{a 1} \sim W N\left(0, \sigma_{e}^{a 1}\right),
$$

then, for the covariance equality constraint in subsection 6.2 to hold, it must be $f_{1}^{a}=(c-2) / 4-\sqrt{(c-2)^{2} / 16-1}, \sigma_{e}^{a}=\gamma_{2} / f_{1}^{a}$. 
If instead we choose the order equal to 2, i.e.,

$$
\text { a2) }\left(1-g_{1}^{2} L^{2}\right) x_{t}=\left(1+f_{1}^{a 2} L\right)\left(1+f_{2}^{a 2} L^{2}\right) e_{t}^{a 2}, \quad e_{t}^{a 2} \sim \text { i.i.d. }\left(0, \sigma_{e}^{a 2}\right) \text {, }
$$

then, the following constraints have to be valid:

$$
\begin{gathered}
{\left[1+\left(1+f_{1}^{a 2}+f_{2}^{a 2}\right)^{2}+\left(f_{1}^{a 2}+f_{2}^{a 2}+f_{1}^{a 2} f_{2}^{a 2}\right)^{2}+\right.} \\
\left.+\left(f_{1}^{a 2}\right)^{2}\left(f_{2}^{a 2}\right)^{2}\right] \sigma_{e}^{a 2}=\gamma_{0} \\
{\left[\left(1+f_{1}^{a 2}+f_{2}^{a 2}\right) f_{1}^{a 2} f_{2}^{a 2}+\left(f_{1}^{a 2}+f_{2}^{a 2}+f_{1}^{a 2} f_{2}^{a 2}\right)\right] \sigma_{e}^{a 2}=\gamma_{2} .}
\end{gathered}
$$

This is a system of two equations in three unknowns so that, in general, infinite solutions are admissible. For example, the system can be solved for $f_{1}^{a 2}$ and $\sigma_{e}^{a 2}$ as functions of $f_{2}^{a 2}$. In this case, it is obtained:

$$
\begin{gathered}
f_{1}^{a 2}=c\left(1+\left(f_{2}^{a 2}\right)^{2}\right) / 2-f_{2}^{a 2}\left(2+f_{2}^{a 2}\right)+ \\
-\left\{\left[c\left(1+\left(f_{2}^{a 2}\right)^{2}\right) / 2-f_{2}^{a 2}\left(2+f_{2}^{a 2}\right)\right]^{2}-\left(2+2\left(f_{2}^{a 2}\right)^{2}+\right.\right. \\
\left.\left.-f_{2}^{a 2} c\right)\left(2+2\left(f_{2}^{a 2}\right)^{2}-f_{2}^{a 2} c+2 f_{2}^{a 2}\right)\right\}^{1 / 2} \\
\sigma_{e}^{a 2}=\gamma_{2} /\left(1+f_{1}^{a 2}+f_{2}^{a 2}\right) f_{1}^{a 2} f_{2}^{a 2}+\left(f_{1}^{a 2}+f_{2}^{a 2}+f_{1}^{a 2} f_{2}^{a 2}\right) .
\end{gathered}
$$

Similarly, if the order of the MA component is assumed to be equal to 3 , the constraint in subsection 6.2 determines a system of 3 equations in 4 unknowns, and the indeterminacy persists when increasing the order.

In the case of the AR component (30b), the choice $f=0$ already generates an aggregate $\operatorname{ARMA}(1,1)$ model but the covariance equality constraint cannot be satisfied. Hence, it must be $f \geq 1$. When $f=1$, the correct MA component is recovered, while for $f>1$ the indeterminacy problem arises. For the AR component (30c) similar results are obtained, $f=0$ is not admissible, when $f=1$ the MA component can be exactly determined, while for $f>1$ infinite solutions are possible.

The sufficient condition for exact identification in Proposition 9 would lead to the choice of the correct model in this example. This would not be the case if, e.g., the generating model were (31).

In summary, even if the processes are restricted to those in the ARIMA class, an infinite number of them is in general compatible with a given aggregated model for $y$. This result depends on the under identification of the MA component and, if its exact identification is achieved 
by means of a priori restrictions, the class of potential disaggregated processes shrinks to contain a finite number of elements. Further a priori restrictions are required to select one element in this class. As an alternative, subjective probabilistic statements about the validity of the restrictions can be expressed, and the formula in (6) used to determine $\widehat{X}_{M}^{*}$.

\section{Conclusions}

In this paper we have suggested a procedure to linearly recover disaggregated values of a time series from the available aggregated values. It minimizes the mean squared disaggregation error and satisfies the compatibility constraint with aggregated values, without requiring assumptions of normality or linearity of the underlying disaggregated stochastic process. When such hypotheses are introduced, the procedure is related to many other disaggregation methods which have been suggested in the literature.

The basic procedure can be extended to estimate many series, possibly at several disaggregated frequencies, or to revise preliminary estimates of disaggregated values. It is so flexible that it can be also adapted to recover missing observations, outliers, linearly disaggregated series, to forecasting, and to deal with uncertainty about the disaggregated process.

The latter issue seems to be important, even if it is rather neglected in the literature. Actually, we have also derived the set of unobservable disaggregated second moments or generating mechanisms which are compatible with their observable aggregated counterparts, and are required for the implementation of disaggregation methods. This set usually contains an infinite number of elements so that particular a priori restrictions have to be imposed for the disaggregated characteristics to be exactly identifiable from the aggregated ones. As an alternative, a probabilistic statement can be made on their relative likelihood, and it is incorporated into the disaggregation procedure. 
The presence of subjective decisions in the implementation of disaggregation procedures is rather unsatisfactory. Unfortunately, it cannot be eliminated. This suggests that the existing effort to increase the frequency of data collection in order for it to match that of the original process, or at least that of usual interest, is really valuable. 


\section{References}

[1] Anderson, O.D. (1974), "An Improved Approach to Inverting the Autocovariance Matrix of a General Mixed Autoregressive Moving Average Time Process", Australian Statistical Journal, 18, pp. 7375.

[2] Boot, J.C.G., Feibes, W. and Lisman, J.H.C. (1967), "Further Methods of Derivation of Quarterly Figures from Annual Data", Applied Statistics, 16, pp. 65-75.

[3] Brewer, K.R.W. (1973), "Some Consequences of Temporal Aggregation and Systematic Sampling for ARMA and ARMAX models", Journal of Econometrics, 1, pp. 133-154.

[4] Brockwell, P.J. and Davis, R.A. (1991), "Time Series: Theory and Methods", Berlin: Springer Verlag.

[5] Chan, W.S. (1993), "Disaggregation of Annual Time-Series Data into Quarterly Figures: A Comparative Study", Journal of Forecasting, 12, pp. 677-688.

[6] Chang, I., Tiao, G.C. and Chen, Ch. (1988), "Estimation of Time Series Parameters in the Presence of Outliers", Technometrics, 30, pp. 193-204.

[7] Chow, G.C. and Lin, A. (1971), "Best Linear Unbiased Interpolation, Distribution and Extrapolation of Time-Series by Related Series", Review of Economics and Statistics, 53, pp. 372-375.

[8] Clements, M.P. and Hendry, D.F. (1993), "On the Limitations of Comparing Mean Squared Forecast Errors", Journal of Forecasting, 12, pp. 617-637.

[9] Cohen, K.J., Müller, W. and Padberg, M.W. (1971), "Autoregressive Approaches to Disaggregation of Time Series Data", Applied Statistics, 20, pp. 119-129. 
[10] Denton, F. (1971), "Adjustment of Monthly or Quarterly Series to Annual Totals: An Approach Based on Quadratic Minimization", Journal of the American Statistical Association, 66, pp. 99-101.

[11] Di Fonzo, T. (1990), "The Estimation of M Disaggregate Time Series when Contemporaneous and Temporal Aggregates Are Known", Review of Economics and Statistics, pp. 178-182.

[12] Fernandez, R.B. (1981), "A Methodological Note on the Estimation of Time-Series", Review of Economics and Statistics, 63, pp. 471476.

[13] Gomez, V. and Maravall, A. (1994), "Estimation, Prediction and Interpolation for Non Stationary Time Series with the Kalman Filter", Journal of the American Statistical Association, 89, pp. 611-624.

[14] Guerrero, V.M. (1990), "Temporal Disaggregation of Time-Series: An ARIMA-Based Approach", International Statistical Review, 58, pp. 29-46.

[15] Harvey, A.C. and Pierse, R.G. (1984), "Estimating Missing Observations in Economic Time Series", Journal of the American Statistical Association, 79, pp. 125-131.

[16] Kato, A.J. (1984), "A Characterization of the Inverse Autocorrelation Function", Communications in Statistics, Theory and Methods, 13, pp. 2503-2510.

[17] Kohn, R. and Ansley, C.F. (1986), "Estimation, Prediction and Interpolation for ARIMA models with Missing Data", Journal of the American Statistical Association, 81, pp. 751-761.

[18] Lisman J.H.C. and Sandee, J. (1964), "Derivation of Quarterly Figures from Annual Data", Applied Statistics, 13, pp. 87-90.

[19] Litterman, R.B. (1983), "A Random Walk, Markov Model for the Distribution of Time Series", Journal of Business and Economic Statistics, 1, pp. 169-173. 
[20] Marcellino, M. (1996), "Some Temporal Aggregation Issues in Empirical Analysis", W.P. UCSD 96/39.

[21] Nijman, T.E. and Palm, F.C. (1986), The Construction and Use of Approximations for Missing Quarterly Observations: A ModelBased Approach", Journal of Business and Economic Statistics, 4, pp. 47-58.

[22] Pena, D. and Maravall, A. (1991), "Interpolation, Outliers and Inverse Auotcorrelations", Communications in Statistics, Theory and Methods, 20, pp. 3175-3186.

[23] Rossi, N. (1982), "A Note on the Estimation of Disaggregate Time Series when the Aggregate is Known", Review of Economics and Statistics, pp. 695-696.

[24] Stram, D.O. and Wei, W.W.S. (1986), "A Methodological Note on Disaggregation of Time Series Totals", Journal of Time Series Analysis, 7, pp. 293-302.

[25] Wei, W.W.S. (1981), "Effects of systematic sampling on ARIMA models", Communications in Statistical-Theoretical Mathematics, 10, pp. 2389-2398.

[26] Wei, W.W.S. and Stram, D.O. (1990), "Disaggregation of Time Series Models", Journal of the Royal Statistical Society, Series B, 52, pp. 453-467.

[27] Weiss, A.A. (1984), "Systematic Sampling and Temporal Aggregation in Time Series Models", Journal of Econometrics, pp. 271-281.

[28] Whittle, P. (1963), "Prediction and Interpolation by Linear Least Squares Methods", London: English Univerisities Press. 

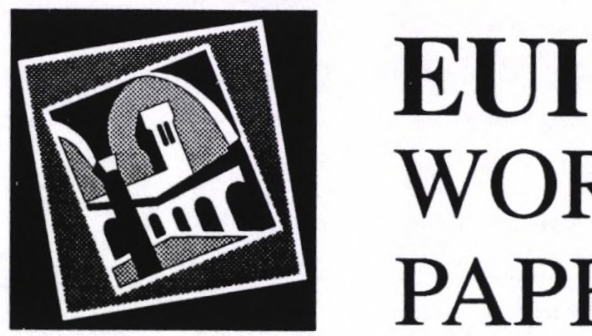

WORKING

PAPERS

EUI Working Papers are published and distributed by the European University Institute, Florence

Copies can be obtained free of charge - depending on the availability of stocks - from:

The Publications Officer

European University Institute

Badia Fiesolana

I-50016 San Domenico di Fiesole (FI)

Italy 


\title{
Publications of the European University Institute
}

To

\author{
The Publications Officer \\ European University Institute \\ Badia Fiesolana \\ I-50016 San Domenico di Fiesole (FI) - Italy \\ Telefax No: $+39 / 55 / 4685636$ \\ e-mail: publish@datacomm.iue.it \\ http://www.iue.it
}

From

Name

Address.

$\square$ Please send me a complete list of EUI Working Papers

$\square$ Please send me a complete list of EUI book publications

$\square$ Please send me the EUI brochure Academic Year 1998/99

Please send me the following EUI Working Paper(s):

No, Author

Title:

No, Author

Title:

No, Author

Title:

No, Author

Title:

Date

Signature 


\section{Working Papers of the Department of Economics Published since 1996}

ECO No. 96/1

Ana Rute CARDOSO

Earnings Inequality in Portugal: High and Rising?

\section{ECO No. $96 / 2$}

Ana Rute CARDOSO

Workers or Employers: Who is Shaping Wage Inequality?

\section{ECO No. $96 / 3$}

David F. HENDRY/Grayham E. MIZON

The Influence of A.W.H. Phillips on

Econometrics

ECO No. 96/4

Andrzej BANIAK

The Multimarket Labour-Managed Firm and the Effects of Devaluation

\section{ECO No. $96 / 5$}

Luca ANDERLINI/Hamid

SABOURIAN

The Evolution of Algorithmic Learning:

A Global Stability Result

ECO No. $96 / 6$

James DOW

Arbitrage, Hedging, and Financial

Innovation

\section{ECO No. 96/7}

Marion KOHLER

Coalitions in International Monetary

Policy Games

\section{ECO No. 96/8}

John MICKLEWRIGHT/ Gyula NAGY A Follow-Up Survey of Unemployment Insurance Exhausters in Hungary

ECO No. 96/9

Alastair McAULEY/John

MICKLEWRIGHT/Aline COUDOUEL

Transfers and Exchange Between

Households in Central Asia

ECO No. 96/10

Christian BELZIL/Xuelin ZHANG

Young Children and the Search Costs of

Unemployed Females
ECO No. 96/11

Christian BELZIL

Contiguous Duration Dependence and

Nonstationarity in Job Search: Some

Reduced-Form Estimates

ECO No. 96/12

Ramon MARIMON

Learning from Learning in Economics

ECO No. $96 / 13$

Luisa ZANFORLIN

Technological Diffusion, Learning and Economic Performance: An Empirical Investigation on an Extended Set of Countries

ECO No. 96/14

Humberto LÓPEZ/Eva ORTEGA/Angel UBIDE

Explaining the Dynamics of Spanish Unemployment

ECO No. 96/15

Spyros VASSILAKIS

Accelerating New Product Development by Overcoming Complexity Constraints

ECO No. 96/16

Andrew LEWIS

On Technological Differences in

Oligopolistic Industries

ECO No. 96/17

Christian BELZIL

Employment Reallocation, Wages and the Allocation of Workers Between

Expanding and Declining Firms

ECO No. 96/18

Christian BELZIL/Xuelin ZHANG

Unemployment, Search and the Gender

Wage Gap: A Structural Model

ECO No. 96/19

Christian BELZIL

The Dynamics of Female Time Allocation upon a First Birth

ECO No. 96/20

Hans-Theo NORMANN

Endogenous Timing in a Duopoly Model with Incomplete Information 
ECO No. 96/21

Ramon MARIMON/Fabrizio ZILIBOTTI 'Actual' Versus 'Virtual' Employment in Europe: Is Spain Different?

ECO No. 96/22

Chiara MONFARDINI

Estimating Stochastic Volatility Models

Through Indirect Inference

ECO No. 96/23

Luisa ZANFORLIN

Technological Diffusion, Learning and

Growth: An Empirical Investigation of a

Set of Developing Countries

ECO No. 96/24

Luisa ZANFORLIN

Technological Assimilation, Trade

Patterns and Growth: An Empirical

Investigation of a Set of Developing

Countries

ECO No. $96 / 25$

Giampiero M.GALLO/Massimiliano

MARCELLINO

In Plato's Cave: Sharpening the Shadows

of Monetary Announcements

ECO No. 96/26

Dimitrios SIDERIS

The Wage-Price Spiral in Greece: An

Application of the LSE Methodology in

Systems of Nonstationary Variables

ECO No. 96/27

Andrei SAVKOV

The Optimal Sequence of Privatization in

Transitional Economies

ECO No. 96/28

Jacob LUNDQUIST/Dorte VERNER

Optimal Allocation of Foreign Debt

Solved by a Multivariate GARCH Model

Applied to Danish Data

ECO No. 96/29

Dorte VERNER

The Brazilian Growth Experience in the

Light of Old and New Growth Theories

ECO No. $96 / 30$

Steffen HÖRNIG/Andrea LOFARO/

Louis PHLIPS

How Much to Collude Without Being

Detected
ECO No. 96/31

Angel J. UBIDE

The International Transmission of Shocks in an Imperfectly Competitive

International Business Cycle Model

ECO No. $96 / 32$

Humberto LOPEZ/Angel J. UBIDE

Demand, Supply, and Animal Spirits

ECO No. 96/33

Andrea LOFARO

On the Efficiency of Bertrand and

Cournot Competition with Incomplete

Information

ECO No. 96/34

Anindya BANERJEE/David F.

HENDRY/Grayham E. MIZON

The Econometric Analysis of Economic

Policy

ECO No. 96/35

Christian SCHLUTER

On the Non-Stationarity of German

Income Mobility (and Some Observations on Poverty Dynamics)

ECO No. $96 / 36$

Jian-Ming ZHOU

Proposals for Land Consolidation and

Expansion in Japan

ECO No. 96/37

Susana GARCIA CERVERO

Skill Differentials in the Long and in the

Short Run. A 4-Digit SIC Level U.S.

Manufacturing Study

\section{米米米}

ECO No. 97/1

Jonathan SIMON

The Expected Value of Lotto when not all

Numbers are Equal

ECO No. 97/2

Bernhard WINKLER

Of Sticks and Carrots: Incentives and the

Maastricht Road to EMU

ECO No. 97/3

James DOW/Rohit RAHI

Informed Trading, Investment, and Welfare 
ECO No. 97/4

Sandrine LABORY

Signalling Aspects of Managers'

Incentives

ECO No. 97/5

Humberto LÓPEZ/Eva ORTEGAVAngel

UBIDE

Dating and Forecasting the Spanish

Business Cycle

ECO No. 97/6

Yadira GONZÁLEZ de LARA

Changes in Information and Optimal Debt

Contracts: The Sea Loan

ECO No. $97 / 7$

Sandrine LABORY

Organisational Dimensions of Innovation

ECO No. 97/8

Sandrine LABORY

Firm Structure and Market Structure: A

Case Study of the Car Industry

ECO No. 97/9

Elena BARDASI/Chiara MONFARDINI

The Choice of the Working Sector in

Italy: A Trivariate Probit Analysis

ECO No. 97/10

Bernhard WINKLER

Coordinating European Monetary Union

ECO No. 97/11

Alessandra PELLONI/Robert

WALDMANN

Stability Properties in a Growth Model

ECO No. 97/12

Alessandra PELLONI/Robert

WALDMANN

Can Waste Improve Welfare?

ECO No. 97/13

Christian DUSTMANN/Arthur van

SOEST

Public and Private Sector Wages of Male

Workers in Germany

ECO No. 97/14

Søren JOHANSEN

Mathematical and Statistical Modelling of

Cointegration
ECO No. 97/15

Tom ENGSTED/Søren JOHANSEN

Granger's Representation Theorem and

Multicointegration

ECO No. 97/16

Søren JOHANSEN/

Ernst SCHAUMBURG

Likelihood Analysis of Seasonal

Cointegration

ECO No. 97/17

Maozu LU/Grayham E. MIZON

Mutual Encompassing and Model

Equivalence

ECO No. 97/18

Dimitrios SIDERIS

Multilateral Versus Bilateral Testing for

Long Run Purchasing Power Parity: A

Cointegration Analysis for the Greek

Drachma

ECO No. 97/19

Bruno VERSAEVEL

Production and Organizational

Capabilities

ECO No. 97/20

Chiara MONFARDINI

An Application of Cox's Non-Nested

Test to Trinomial Logit and Probit

Models

ECO No. 97/21

James DOW/Rohit RAHI

Should Speculators be Taxed?

ECO No. 97/22

Kitty STEWART

Are Intergovernmental Transfers in

Russia Equalizing?

ECO No. 97/23

Paolo VITALE

Speculative Noise Trading and

Manipulation in the Foreign Exchange

Market

ECO No. 97/24

Günther REHME

Economic Growth, (Re-)Distributive

Policies, Capital Mobility and Tax

Competition in Open Economies 
19EO No. 97/25

SUSAna GARCIA CERVERO

A Historical Approach to American Skill

Differentials

ECO No. 97/26

Susana GARCIA CERVERO

Growth, Technology and Inequality: An Industrial Approach

ECO No. 97/27

Bauke VISSER

Organizational Structure and Performance

ECO No. 97/28

Pompeo DELLA POSTA

Central Bank Independence and Public

Debt Convergence in an Open Economy

Dynamic Game

ECO No. 97/29

Matthias BRUECKNER

Voting and Decisions in the ECB

ECO No. 97/30

Massimiliano MARCELLINO

Temporal Disaggregation, Missing

Observations, Outliers, and Forecasting:

A Unifying Non-Model Based Procedure 
